J. Dairy Sci. 96:2201-2213

http://dx.doi.org/10.3168/jds.2012-6036

(C) American Dairy Science Association ${ }^{\circledR}, 2013$.

\title{
Feed restriction, but not L-carnitine infusion, alters the liver transcriptome by inhibiting sterol synthesis and mitochondrial oxidative phosphorylation and increasing gluconeogenesis in mid-lactation dairy cows
}

\author{
H. Akbar, ${ }^{*}$ M. Bionaz,† D. B. Carlson, ${ }^{* 1}$ S. L. Rodriguez-Zas, ${ }^{*}$ R. E. Everts, ${ }^{* 2}$ H. A. Lewin, ${ }^{*}$ J. K. Drackley, ${ }^{*} \ddagger$ \\ and J. J. Loor ${ }^{*} \ddagger^{3}$ \\ *Department of Animal Sciences, University of Illinois, Urbana 61801 \\ †Department of Animal and Rangeland Sciences, Oregon State University, Corvallis 97331 \\ ‡Division of Nutritional Sciences, University of Illinois, Urbana 61801
}

\begin{abstract}
Abomasal carnitine infusion during acute feed restriction increases hepatic fatty acid oxidation and decreases liver lipid in dairy cows. Eight mid-lactation Holstein cows were used in a replicated $4 \times 4$ Latin square design with 14 -d periods. A $2 \times 2$ factorial arrangement was used to determine the effects of water infusion + ad libitum dry matter intake (DMI), water infusion + restricted DMI (50\% of previous 5-d average), L-carnitine infusion $(20 \mathrm{~g} / \mathrm{d})+$ ad libitum DMI, or L-carnitine infusion + restricted DMI. Liver RNA from 7 healthy cows was used for transcriptome profiling using a bovine microarray. An ANOVA with a false discovery rate was used to identify treatment and interaction effects. A substantial transcriptome change was observed only with DMI restriction, resulting in 312 (155 downregulated, 157 upregulated) differentially expressed genes. Quantitative PCR was performed to verify microarray data and measure expression of additional genes not present on the microarray. The quantitative PCR data confirmed the effect of feed restriction but not of L-carnitine treatment. Feed restriction increased expression of GPX3 and of genes associated with gluconeogenesis $(P C, P D K 4)$, inflammation (SAA3), and signaling (ADIPOR2). In contrast, feed restriction downregulated BBOX, a key for L-carnitine biosynthesis, and the transcription factor HNF4A. The bioinformatics functional analysis of genes affected by DMI restriction uncovered biosynthesis of cholesterol and energy generation by mitochondrial respiration as the most relevant and inhibited functions. The data also indicated an increase of flux toward gluconeogenesis. We interpreted those results as a likely response
\end{abstract}

Received August 6, 2012.

Accepted January 2, 2013.

${ }^{1}$ Current address: Elanco Animal Health, Greenfield, IN 46140.

${ }^{2}$ Current address: Sequenom Inc., San Diego, CA 92121.

${ }^{3}$ Corresponding author: jloor@illinois.edu of the liver to spare energy and provide glucose for the lactating mammary gland during feed deprivation.

Key words: L-carnitine, feed restriction/negative energy balance, hepatic gene profile, dairy cow

\section{INTRODUCTION}

Metabolic adaptations to negative energy balance (NEB) in dairy cows are integral for a successful transition from gestation to lactation. Failure to adapt to NEB is the basis for many metabolic disorders and infectious diseases commonly afflicting dairy cows (Drackley et al., 2005; Bertoni et al., 2009). The liver orchestrates flux and interconversion of nutrients and metabolites to support the dramatic changes in the supply of, and demand for, nutrients during the transition period. Alterations in gene expression, enzyme activities, and the resultant nutrient fluxes are essential for optimal liver function and nutrient interconversion (Reynolds et al., 2003; Dann and Drackley, 2005; Loor et al., 2005).

Fatty liver in dairy cows and humans is associated with several negative effects; therefore, altering both the degree of adipose tissue deposition and the hepatic capacity for long-chain FA oxidation are of considerable interest (Bobe et al., 2004; Reddy and Rao, 2006). The activity and expression of carnitine palmitoyltransferase-I (CPT-I), the mitochondrial enzyme for which L-carnitine serves as a cofactor, increases around parturition to facilitate oxidation of mobilized NEFA (Drackley et al., 2005; Loor et al., 2005). Supplemental L-carnitine has shown potential to decrease or prevent liver triacylglycerol (TAG) accumulation in feedrestricted dairy cows (Carlson et al., 2006) and laboratory animals (Bykov et al., 2003; Spaniol et al., 2003).

Functional genomics approaches using microarray technology to allow for mechanistic inferences of tissue metabolic responses. Previous research with a cattlespecific cDNA microarray identified several key adaptations to NEB in the liver of periparturient dairy cows (Loor et al., 2005, 2006). Although feed restriction of 
lactating cows may not mimic the normal decrease in voluntary feed intake of late-pregnant cows, this model has been valuable as a tool for understanding the effect of negative nutrient balance on liver molecular adaptations (Bradford and Allen, 2005; Velez and Donkin, 2005) without the confounding effect of the hormonal environment that characterizes the peripartum period. Therefore, the main objective of this study was to determine the extent to which abomasal L-carnitine infusion and acute DMI restriction alter gene expression patterns in liver and how those alterations are linked with in vitro rates of lipid metabolism. Other portions of data from the animals used for the current study have already been reported (Carlson et al., 2006).

\section{MATERIALS AND METHODS}

\section{Experimental Design and Treatments}

Samples were obtained from 8 mid-lactation Holstein cows (132 \pm 36 DIM) from a previous experiment (Carlson et al., 2006). The cows were fitted with ruminal cannulas and used in a replicated $4 \times 4$ Latin square with 14-d periods as previously described (Carlson et al., 2006, 2007b). None of the selected cows exhibited signs of clinical conditions. Briefly, treatments were factorial combinations of abomasal infusion and amount of DMI: water infusion + ad libitum DMI, water infusion + restricted DMI, carnitine infusion + ad libitum DMI, carnitine infusion + restricted DMI. Each 14-d period consisted of a washout period (d 1-4) during which all cows were infused with water, whereas infusion treatments began on d 5 and continued until d 14. Infusions were conducted every $6 \mathrm{~h}$; cows assigned to the L-carnitine and ad libitum DMI and L-carnitine and restricted DMI treatments received $20 \mathrm{~g} / \mathrm{d}$ of L-carnitine (Lonza Inc., Allendale, NJ) postruminally. On d 10 of each period, DMI was restricted to $50 \%$ of the previous 5 -d average DMI for each cow on the restricted water and L-carnitine treatments. Cows were housed in individual tie stalls, milked twice daily, and fed a diet that was balanced to meet the nutrient requirements of Holstein cows in lactation, as previously reported (Carlson et al., 2006).

\section{Liver Tissue Collection and RNA Extraction}

Liver tissue $(\sim 3-6 \mathrm{~g})$ was obtained by puncture biopsy (Loor et al., 2005) under local anesthesia (2\% lidocaine $\mathrm{HCl}$ ) on d 14 of each period. Approximately 0.2 to 0.3 $\mathrm{g}$ of liver tissue was immediately placed in ice-cold TRIzol reagent (Cat. No. 15596-026, Invitrogen, Grand Island, NY) and homogenized. Samples were stored at $-80^{\circ} \mathrm{C}$ until RNA extraction as described previously
(Loor et al., 2005, 2006). Genomic DNA was removed from RNA with RNase-free DNase (Cat. No. 79254, Qiagen, Valencia, CA) using RNeasy Mini Kit columns (Cat. No. 74104, Qiagen). The RNA concentration was measured using a NanoDrop ND-1000 spectrophotometer (NanoDrop Technologies, Wilmington, DE), and RNA quality was assessed using a 2100 Bioanalyzer (Agilent Technologies Inc., Santa Clara, CA). The average RNA integrity number value for samples was $8.0 \pm$ 0.4 . The extracted and cleaned RNA was resuspended in RNase free water (Cat No. 74104, Qiagen) and stored at $-80^{\circ} \mathrm{C}$ until microarray or quantitative PCR (qPCR) analysis. From a preliminary examination of the liver microarray data it appeared that one cow had a substantial inflammatory condition throughout the study (e.g., large upregulation of SAA3); therefore, we removed that cow from all the analyses.

\section{Liver Tissue Composition and Metabolism}

Protocol and results of liver tissue composition and in vitro metabolism were reported previously (Carlson et al., 2006). Because only 7 out of 8 cows were used for gene expression analysis in the present manuscript, the data from tissue and metabolism analysis were reanalyzed and results are reported in Figure 1.

\section{Microarrays, qPCR Analysis, and Microarray Data Analysis}

These details can be found in Supplemental Table S1 (available online at http://www.journalofdairyscience. org/), which contains primer sequence information. The microarray data presented in this manuscript have been deposited at the National Center for Biotechnology Information's (NCBI) Gene Expression Omnibus and are accessible through GEO series accession number GSE43307.

\section{Statistical Analysis}

Differences in relative expression ratios were deemed significant at an overall infusion $\times$ DMI false discovery rate (FDR) cutoff of $P \leq 0.15$ (equivalent to uncorrected $P \leq 0.05$ ). The complete list of genes affected at this threshold is provided in Supplemental Table S2 (available online at http://www.journalofdairyscience. org/). For qPCR data, normalized values were fitted to a mixed-effects model and analyzed using Proc MIXED of SAS (SAS Institute Inc., Cary, NC). Fixed effects in the model were infusion type, amount of DMI, and associated interaction. Random effects were cow nested within square and period nested within square. Significance of the $F$-test of fixed effects was declared at $P$ 

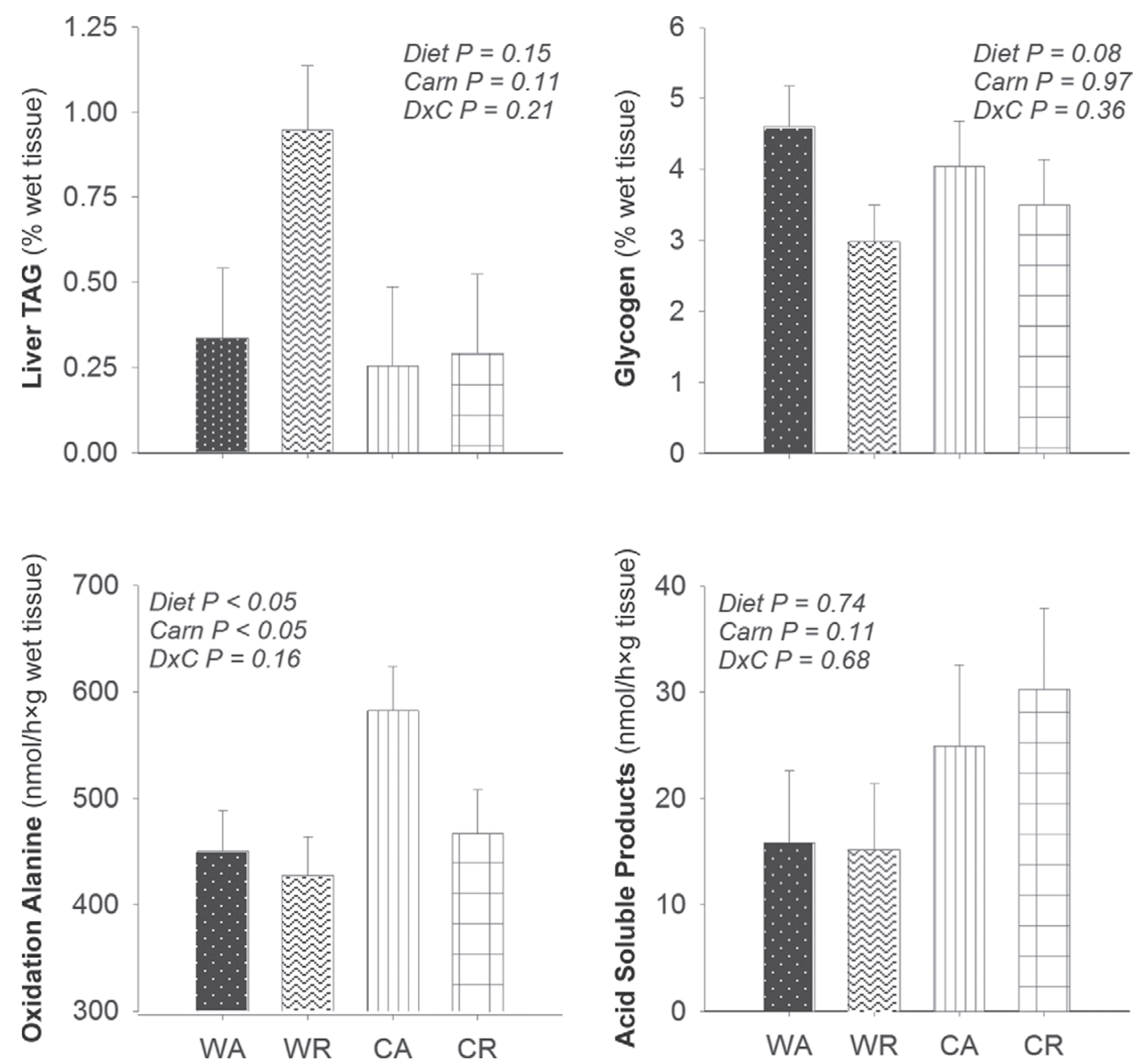

Figure 1. Liver concentration of triaclyglycerol (TAG) and glycogen and magnitude of alanine oxidation in vitro and output of acid-soluble products from palmitate (mainly BHBA) in mid-lactating Holstein cows receiving an abomasal water infusion and ad libitum DMI (WA), water infusion and 50\% DMI restriction (WR), L-carnitine infusion $(20 \mathrm{~g} / \mathrm{d})$ and ad libitum DMI (CA), or L-carnitine infusion (20 g/d) and 50\% DMI restriction (CR). Diet (D; ad libitum or feed restricted) and carnitine (Carn, C) effects and interactions are shown.

$\leq 0.05$, with tendencies discussed at $0.05<P \leq 0.15$. Means associated with a significant interaction $(P \leq$ 0.05) were separated with Tukey's test.

\section{Gene Expression Data Mining}

The entire data set of microarray and qPCR with associated statistical $\mathrm{P}$ values were imported into Ingenuity Pathway Analysis (IPA, http://www.ingenuity. com/; Ingenuity Systems Inc., Redwood City, CA) and Database for Annotation, Visualization and Integrated Discovery (DAVID; Huang et al., 2009) analyses tools (http://david.abcc.ncifcrf.gov; SAIC-Frederick Inc., Frederick, MD). In both tools the complete annotated microarray was used as the reference data set (i.e., background) for enrichment analysis. For IPA analysis, the results from function and pathways analyses were downloaded and provided in Supplemental Tables
S3 and S4 (available online at http://www.journalof dairyscience.org/). Functions and pathways enriched with a $P$-value $\leq 0.001$ were considered significantly overrepresented and, thus, are discussed as biologically significant. For DAVID analysis (Huang et al., 2009), the 3 gene ontology (GO) categories and Kyoto Encyclopedia of Genes and Genomes (KEGG) pathways enriched with a Benjamini-Hochberg FDR $\leq 0.10$ were considered significantly overrepresented and are discussed. More details on the applicability of these tools for the bioinformatics analysis of microarray data have been published previously (Bionaz et al., 2012).

\section{Dynamic Impact Approach}

We have provided functional analysis also using the novel bioinformatics tool direct impact approach (DIA; Bionaz et al., 2012). The DIA is a tool that allows 
visualization of the impact and direction of the impact of differentially expressed genes (DEG) on pathways or biological functions from several annotation databases, including GO and KEGG pathways. The impact and the direction of the impact for KEGG pathways were calculated only for those terms that were represented by at least $30 \%$ in the microarray compared with the entire annotated bovine genome. The redundancy in GO terms in the results from DAVID and DIA analyses was corrected and visualized using reduce and visualize gene ontology (REVIGO; Supek et al., 2011).

\section{Network Analysis}

Networks among DEG were developed using IPA. Connections among genes were based on known relationships available in the IPA knowledge base. To uncover transcription factors most responsible for regulation of expression of DEG, we have added to the network all possible transcription factors (transcription regulators plus nuclear receptors) able to affect expression of the DEG contained in the IPA knowledge base. Genes associated with main functions of DEG in the networks were uncovered using IPA and by manual annotation using the gene annotation at the NCBI web (http:// www.ncbi.nlm.nih.gov/pubmed/); results are shown in Supplemental Figure S1 (available online at http:// www.journalofdairyscience.org/).

\section{RESULTS}

Biochemical results from reanalysis of the subset of cows used in the present study (Figure 1) were consistent with the full data set reported by Carlson et al. (2006). Briefly, DMI restriction numerically increased liver TAG concentration $(P=0.15)$ and decreased $(P$ $=0.08$ ) liver glycogen. L-Carnitine infusion tended $(P$ $=0.11)$ to prevent or decrease TAG accumulation and tended $(P=0.11)$ to increase in vitro conversion of $[1-$ $\left.{ }^{14} \mathrm{C}\right]$ palmitate to acid-soluble products. Feed restriction decreased $(P<0.05)$ the in vitro oxidation of $\left[1-{ }^{14} \mathrm{C}\right]$ alanine, whereas L-carnitine infusion increased $(P<$ 0.05) oxidation of alanine.

\section{Differential Expression of Hepatic Genes Using Microarray}

The analysis revealed that only 1 gene (LOC782945, solute carrier organic anion transporter family, member 2A1-like) was significantly (FDR $\leq 0.15)$ affected by the interaction of feed restriction $\times$ L-carnitine infusion. No genes were significantly affected by L-carnitine alone, whereas feed restriction alone affected expression of 312 genes (157 upregulated and 155 downregulated).
Due to the lack of L-carnitine effect on the transcriptome, the functional analysis of microarray data was performed only on genes significantly affected by feed restriction.

Seventeen DEG with $\geq 1.5$-expression ratio (i.e., $>75 \%$ change in expression) in DMI restriction versus control are shown in Table 1. Among the upregulated genes, the expression of GPX3 involved in the detoxification of hydrogen peroxide was the most induced (the only one with $>2$-fold increase). Other genes upregulated by $\geq 1.5$-fold were associated with several different functions including gluconeogenesis (i.e., pyruvate carboxylase). The genes downregulated by $\geq 1.5$-fold were mostly associated with lipid synthesis, particularly cholesterol synthesis.

\section{Expression of Genes by $q P C R$}

The 5 genes used to verify the microarray data (ADIPOR2, CPT1A, GPX3, PC, and SAA3) confirmed that the observed expression patterns were comparable between the techniques (Figure 2). The $\mathrm{qPCR}$ data confirmed the lack of interaction between DMI restriction and L-carnitine infusion - except for ADIPOR2, $P D K 4$, and $S A A 3$, for which a tendency was observed. In contrast with microarray, the qPCR data revealed a significant downregulation of $C P T 1 A$ by L-carnitine infusion. In addition, ADIPOR2 was significantly affected by DMI restriction in qPCR but was only numerically affected in microarray $(\mathrm{FDR}=0.20$; Supplemental Table S2). Feed restriction upregulated $(P<0.05)$ the expression of $P D K 4$ and $S A A 3$, but downregulated ( $P$ $<0.05) B B O X$ and HNF $4 A$ (Figure 2).

\section{Functional Enrichment of Genes Affected by Feed Restriction}

Functional enrichment analysis with IPA revealed that cellular movement, skeletal and muscular system development and function, cell-to-cell signaling and interaction, respiratory system development and function, tissue development, and lipid metabolism were the most enriched functions (Table 2, Supplemental Table S3). Except for lipid metabolism, where most of the genes were downregulated and deemed to be inhibited, the other functions all appeared to be induced (i.e., mostly composed by upregulated genes; Tables 2 and Supplemental Table S3). Among canonical metabolic pathways, the DEG as a result of DMI restriction significantly $(P<0.01)$ enriched biosynthesis of steroids, synthesis and degradation of ketone bodies, and pyruvate metabolism; in all of those pathways most of the genes were downregulated (Tables 2 and Supplemental Table S4). 


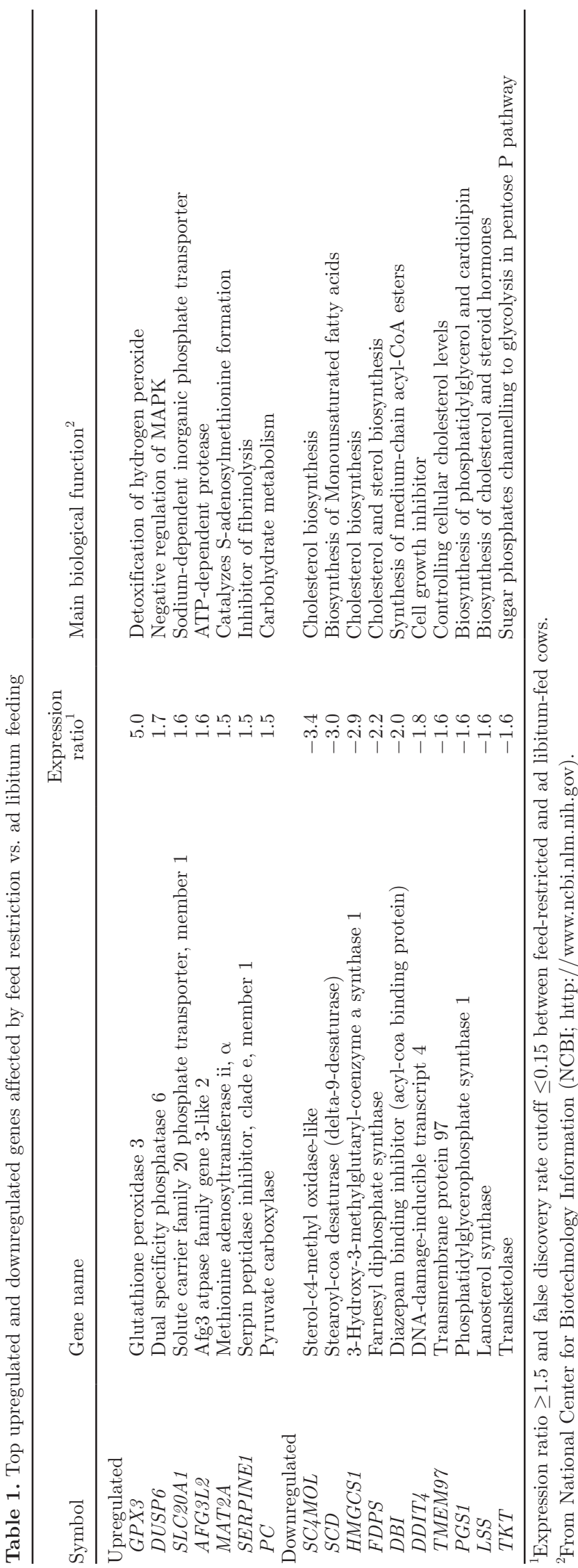

Results of the functional enrichment using DAVID with DEG due to feed restriction highlighted that mitochondrion, mitochondrial inner membrane, respiratory chain, and steroid biosynthesis were the most-enriched terms among downregulated genes (Table 2). When all the terms enriched with an expression analysis systematic explorer (EASE; Hosack et al., 2003) score $<0.10$ were considered, the use of REVIGO suggested that among GO biological process (BP) induced were proliferation and development, cell signaling, and translation (Supplemental Figure S2, available online at http:// www.journalofdairyscience.org/), whereas inhibited processes were sterol metabolism, protein folding, electron transport chain, and response to oxidative stress (Supplemental Figure S3, available online). Using the same analysis for the GO cellular component, no factors appeared to be significantly induced; but the overall analysis suggested an inhibition of mitochondrial inner membrane, proteasome, and envelope components (Supplemental Figure S4, available online).

\section{Functional Categories Affected by Feed Restriction as Uncovered by DIA}

Terms in GO BP, molecular function (MF), and KEGG_PATHWAY with the greatest effect are shown in Table 2. The DIA analysis of GO BP uncovered the most affected and inhibited terms to be those related with cholesterol synthesis, whereas phosphate transport and protein homotetramerization, and inactivation of mitogen-activated protein kinases (MAPK) were activated (Table 2). Among the most affected and inhibited GO MF terms were the ones related with lipid metabolism, particularly sterol biosynthesis (Table 2).

To provide a more holistic view, we used REVIGO to group redundant GO terms (Supplemental Figures S5 and S6, available online at http://www.journalofdairy science.org/). The analysis showed that the epithelial differentiation, inactivation of MAPK, sulfur AA metabolism (also encompassing gluconeogenesis), and phosphate transport (including lipid storage and FA transport) were the most activated GO BP (Supplemental Figure S5). Cholesterol biosynthesis and hair follicle development (encompassing development of several tissues and cell cycle arrest) were the most inhibited GO BP (Supplemental Figure S6). The other inhibited processes were mitochondria outer membrane translocase complex assembly (also encompassing Golgi, peroxisome, and histone deacetylation), electron transport chain, regulation of sodium ion transport, and response to cold (Supplemental Figure S6). Among GO cellular components, the small-subunit processome (including ribosome and translation-related components), desmosome, lipid particles, and endoplasmic reticulum-Golgi 


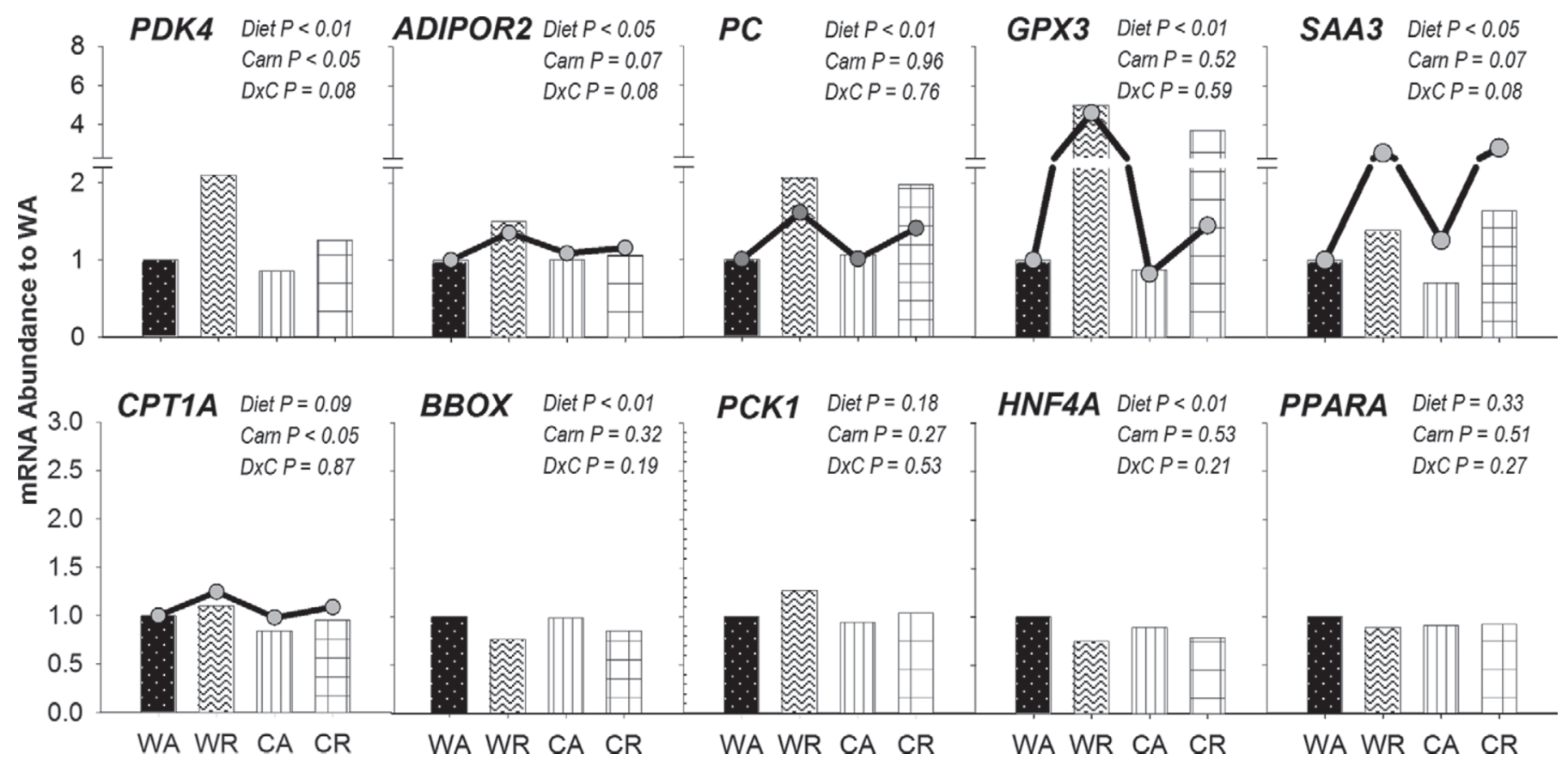

Figure 2. Liver mRNA expression by quantitative PCR (bars) or microarray (lines and symbols) in mid-lactating Holstein cows receiving an abomasal water infusion and ad libitum DMI (WA), water infusion and 50\% DMI restriction (WR), L-carnitine infusion (20 g/d) and ad libitum DMI (CA), or L-carnitine infusion (20 g/d) and 50\% DMI restriction (CR). Statistical information pertains to quantitative PCR data. Diet (D; ad libitum or feed restricted) and carnitine (Carn, C) effects and interactions are shown.

intermediate compartments were the most activated (Supplemental Figure S7, available online); in contrast, transport vesicle, polysome, chaperonin-containing Tcomplex, and respiration chain were among the most inhibited (Supplemental Figure S8, available online).

The DIA analysis of main KEGG pathway categories clearly indicated an overall high effect and inhibition of metabolism by DMI restriction with a very minor effect on other categories (Figure 3). Among metabolism subcategories, the most affected and inhibited were lipid metabolism and metabolism of terpenoids and polyketides, followed by carbohydrates metabolism and amino acid metabolism (Figure 3). The only subcategory that was induced by DMI restriction was metabolism of other AA (Figure 3).

The inhibition of lipid metabolism in DMI-restricted cows was mostly due to an evident inhibition of steroid biosynthesis and synthesis and degradation of ketone bodies, which were the most affected KEGG pathways (Figure 3, Table 2, and Supplemental Figures S9 to S11, available online at http://www.journalofdairyscience. $\mathrm{org} /$ ). For the metabolism of terpenoids and polyketides, the only pathway composing the subcategory in KEGG was the terpenoid backbone biosynthesis, which was highly inhibited by DMI restriction (Table 2, Figures 3 and Supplemental Figure S12, available online).

\section{Network Among DEG}

The interactive network among the DEG due to feed restriction is reported in Supplemental Figure S1. The DEG-forming networks were related to steroid biosynthesis, FA oxidation, gluconeogenesis, cell cycle, apoptosis, protein synthesis, protein folding, oxidative phosphorylation, and electron transport chain. The network revealed a coordinated downregulation of cholesterol synthesis, with a central role for the transcription factor SREBF2 that regulates expression of genes such as HMGCS1, SC4MOL, LSS, FDPS, and $H M G C R$, all of which were markedly downregulated due to DMI restriction (Supplemental Table S2). Other transcription regulators such as PPARA and PPARG were linked with genes important in steroid biosynthesis (SC4MOL, HMGCS1, FDPS), gluconeogenesis (PC, $P D K 4, P C K 1)$, and FA oxidation (CPT1A, ACAA1). Among transcription regulators affected by DMI restriction, BTG1, FOSB, CNOT\%, MAFB, and PCBP4 were all upregulated (Supplemental Figure S1 and Supplemental Table S2) and are putatively regulators of genes associated with several functions, such as cell growth and proliferation, inflammatory response, and oxidative phosphorylation. It is evident from the network in Supplemental Figure S1 that for each function a 
Table 2. Top biological terms from the functional analysis of liver microarray data from mid-lactating Holstein cows receiving an abomasal water infusion and ad libitum DMI (WA), water infusion and 50\% DMI restriction (WR), L-carnitine infusion (20 g/d) and ad libitum DMI $(\mathrm{CA})$, or L-carnitine infusion $(20 \mathrm{~g} / \mathrm{d})$ and $50 \% \mathrm{DMI}$ restriction $(\mathrm{CR})^{1}$

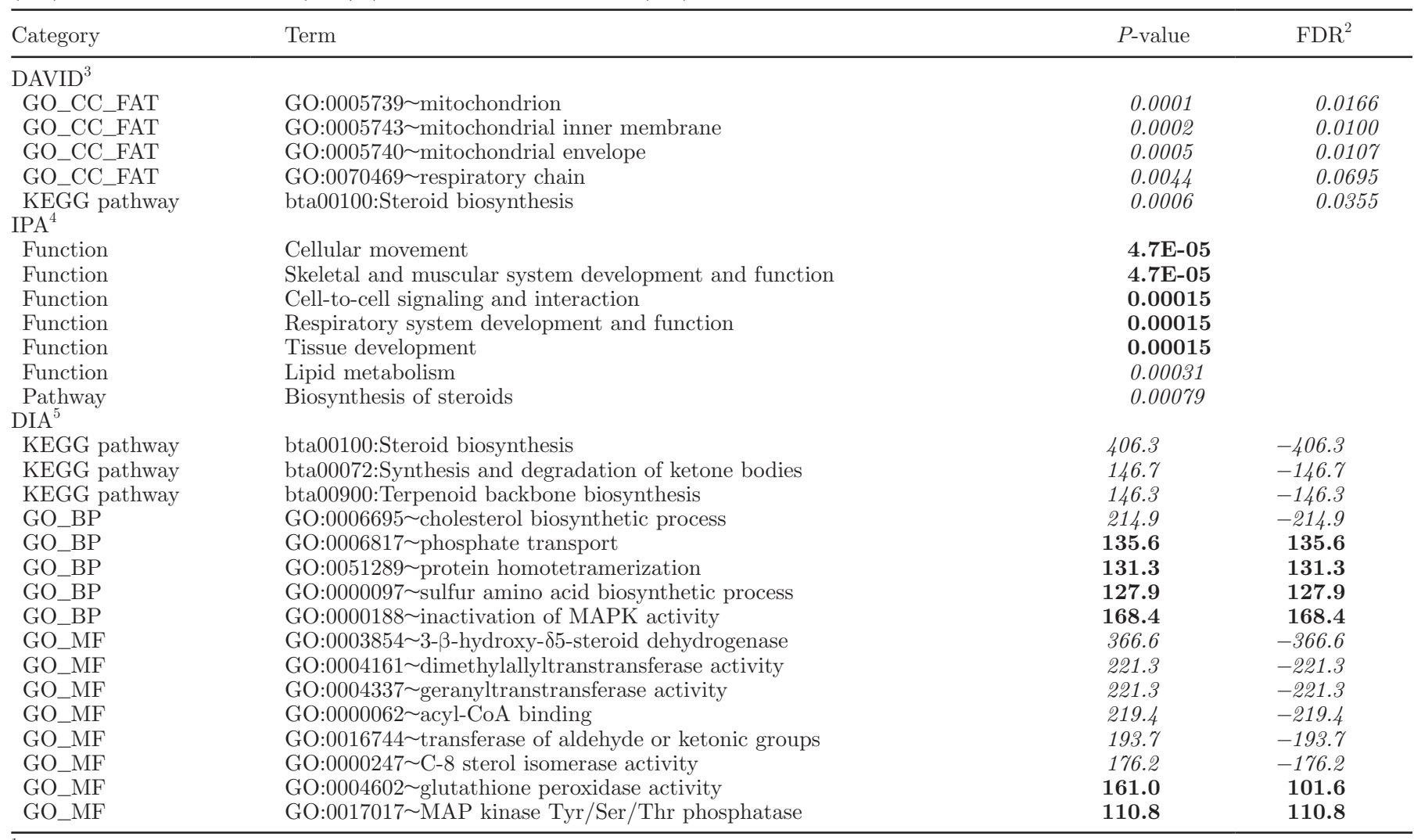

${ }^{1}$ Data shown are a subset (i.e., most significant) of the results obtained using DAVID, Ingenuity Pathway Analysis (IPA), and Dynamic Impact Approach (DIA). P-values or impacts in italic denote terms deemed to be overall inhibited and bold denotes terms deemed to be overall activated.

${ }^{2} \mathrm{FDR}=$ false discovery rate.

${ }^{3}$ Shown are the significantly-enriched terms (FDR <0.10) within the gene ontology (GO) cellular component (CC) categories plus Kyoto Encyclopedia of Genes and Genomes (KEGG) pathways.

${ }^{4}$ Shown are the most significant (uncorrected $P$-value $<0.001$ ) functions and pathways.

${ }^{5}$ Shown are the most affected terms (>100 impact) within the GO categories [biological process (BP) and molecular function (MF)] plus KEGG pathways.

complex transcriptional factor network is coordinately regulating the adaptation to reduced DMI.

\section{DISCUSSION}

The periparturient or transition period is characterized by major hormonal, metabolic, and inflammationrelated changes that seem to be causally associated with reduction in voluntary DMI leading to NEB (Bertoni et al., 2009). To meet the energy requirements for galactopoiesis after parturition, dairy cattle rely on mobilization of adipose and muscle tissue. The period of NEB typically lasts until milk yield starts to decline around 7 to 10 wk after parturition, at which point the energy from the DMI becomes sufficient to meet the cow's requirements (Butler and Smith, 1989). As a consequence of NEB around calving, a gap between nutrient supply and demand exists, which often contributes to the onset of metabolic disorders (Collard et al., 2000).

Data obtained from nonruminants indicates that Lcarnitine supplementation increases hepatic gluconeogenesis (Ji et al., 1996; Owen et al., 2001), with similar responses observed in early postpartal cows (Carlson et al., 2007a). Thus, this nutrient provides benefits to the cow, considering the extreme glucose demand imposed by lactation. The original study, from which tissue used in the present study was derived (Carlson et al., 2006), attempted to model the NEB that occurs during the peripartum period with the caveat that some factors (e.g., hormonal status) preclude a direct comparison of feed restriction in mid-lactation and the peripartum. 


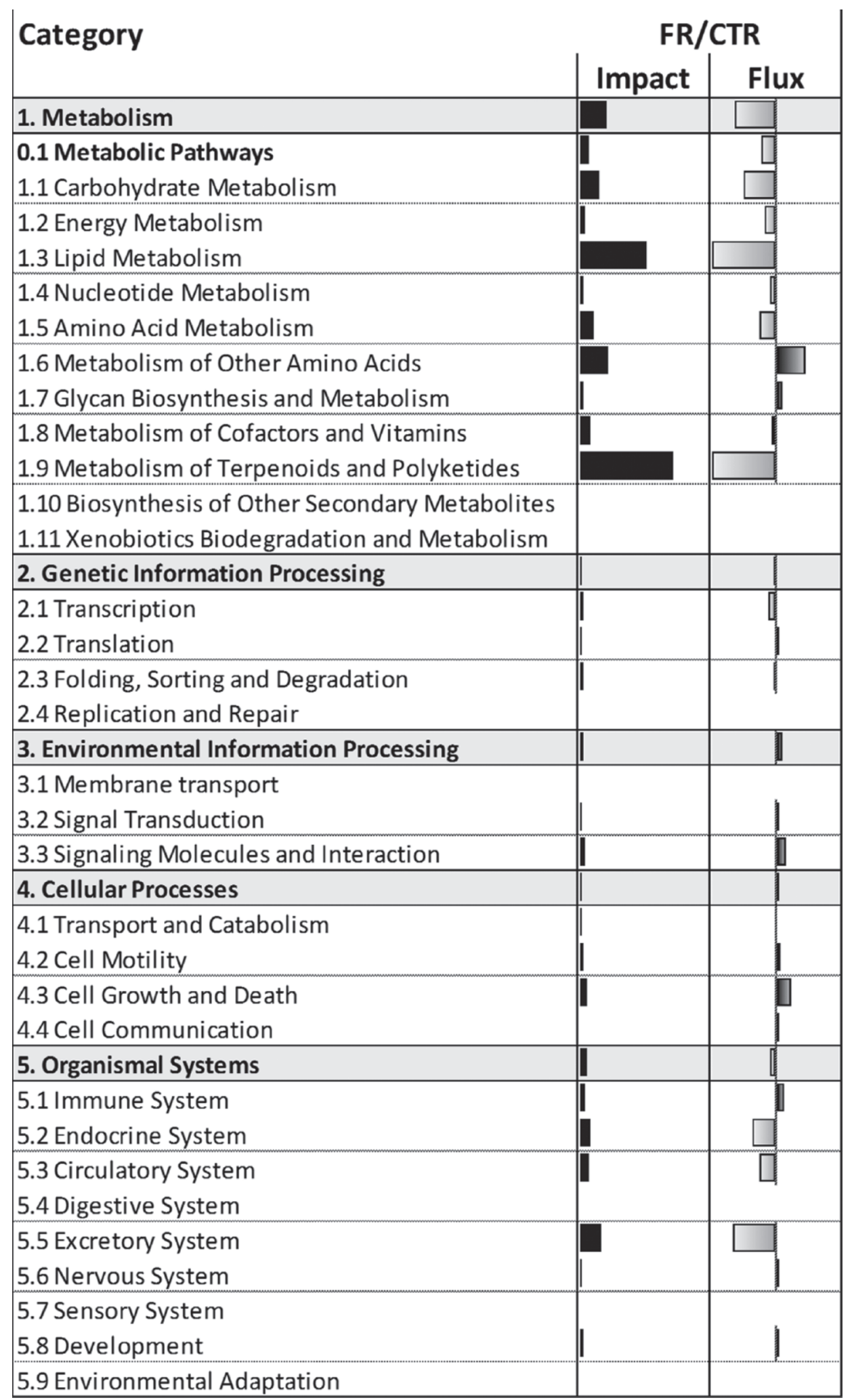

Figure 3. Impact and flux of main Kyoto Encyclopedia of Genes and Genomes pathways categories and subcategories as calculated by the dynamic impact approach of differentially expressed genes (DEG) in mid-lactating Holstein cows receiving restricted (feed restricted, FR) versus control (ad libitum, CTR) DMI. Reported are the total impact (black horizontal bars; the greater the bar the higher the impact) and the direction of the impact (or flux; gray bars expanding left denote inhibition and black bars expanding right denote activation) of DEG on the categories and subcategories. 


\section{Lipid Metabolism During Feed Restriction}

Previous microarray studies in mice (Bauer et al., 2004) and cows (Loor et al., 2007, McCarthy et al., 2010) showed that a state of NEB induces the expression of genes associated with lipid catabolism, whereas it inhibits cholesterol synthesis. The present functional analyses revealed that feed restriction in mid-lactating cows causes a similar effect, including an inhibition of the mitochondrial respiratory chain. Furthermore, DMI restriction appeared to have induced a switch toward energy conservation through the downregulation of energy-generation pathways (e.g., oxidative phosphorylation).

Cholesterol and its derivatives (steroid hormones, bile acids) are essential for vital functions in the body, replenishment of cellular membranes, and absorption of dietary lipid. The liver achieves cholesterol hemostasis in part through the control of expression of the enzymes required for its biosynthesis, and regulates cholesterol turnover rate via lipoprotein metabolism (Cummins et al., 2006). Endogenous synthesis of cholesterol from acetyl-CoA, via a set of key cytosolic enzymes, regulates cholesterol homeostasis in mammals (Viturro et al., 2009). The decrease in cholesterol synthesis by feed restriction suggested by the microarray data in the present experiment is supported by previously observed lower liver and blood cholesterol concentrations in feedrestricted lactating cows compared with cows fed ad libitum (Brumby et al., 1975; Kuhla et al., 2009).

The suggested inhibition of MAPK (Table 2) is interesting, as MAPK are known to be involved in the regulation of steroid production by different mechanisms. Prominent among these mechanisms is the induction of expression of low-density lipoprotein receptor (LDLR; Singh et al., 1999), which is essential for the liver to uptake cholesterol-rich lipoproteins. Thus, MAPK plays a pivotal role in regulating cholesterol homeostasis (Go and Mani, 2012). Our data revealed that the marked inhibition of steroid hormone biosynthesis due to feed restriction might have been partly associated with inactivation of MAPK activity (Table 2), perhaps as a result of upregulation of DUSP6 (Table 1), which can inhibit MAPK (Arkell et al., 2008).

Together, those observations suggest a likely compensation of the inhibition of cholesterol synthesis by the liver via an increased uptake of cholesterol from circulating lipoproteins; however, contrary to such a suggestion, in feed-restricted postpartum dairy cows the expression of $L D L R$ was reduced in the liver (Loor et al., 2007). The suggested inhibition of MAPK in the liver due to feed restriction remains to be verified; however, a relationship between MAPK inhibition and steroid hormone synthesis has been observed in steroido- genic tissues. The process of steroid hormone synthesis is initiated by the cleavage of the cholesterol-side chain to produce pregnenolone, which requires the transport of cholesterol from cytoplasm to the mitochondrial inner cell membrane mediated by the steroidogenic acute regulatory protein (Liu et al., 2006; Hutchinson et al., 2012). The MAPK is essential in inducing steroidogenic acute regulatory protein activity and, thus, steroidogenesis (Poderoso et al., 2009).

The FA metabolism pathway was not overall induced by feed restriction in the present experiment when considering all the DEG (Supplemental Figure S9); however, we cannot exclude an increase in flux of long-chain FA oxidation in our study due to the upsurge in expression of related genes such as ACAA1 and ADIPOR2 and, numerically, of CPT1A (Figure 2 and Supplemental Table S2). As previously discussed (Bionaz and Loor, 2012), the increase in FA oxidation appears to be under the control of available FA and CPT1A activity instead of large transcriptome changes. The combination of the lack of large transcriptomics changes and the in vivo increase of plasma NEFA (Carlson et al., 2006), but no marked change in in vitro FA oxidation (Figure 1) in cows feed-restricted versus ad libitum, supports such a conclusion.

Both CPT-1 activity and CPT1A expression increased in early lactation compared with late pregnancy, and were positively associated with liver TAG and serum NEFA (Dann and Drackley, 2005; Loor et al., 2005). Even though a positive correlation was observed between CPT-1 and liver TAG, moderate nutrient restriction during the dry period was associated with increased CPT-1 activity, increased CPT1A transcript abundance (Loor et al., 2006), and increased hepatic carnitine concentration (Grum et al., 1996); all of which were associated with decreased liver TAG accumulation relative to cows allowed ad libitum nutrient intake throughout the dry period.

Increased hepatic ADIPOR2 expression was associated with greater in vitro hepatic FA oxidation, lower liver TAG, and decreased serum insulin in peripartal dairy cows (Loor et al., 2006). In the present study, however, upregulated ADIPOR2 expression did not coincide with the increase in in vitro long-chain FA oxidation observed in the restricted water treatment (i.e., acid-soluble products; Figure 1) despite a tendency for a positive correlation $(\mathrm{r}=0.37 ; P=0.08)$; in fact, liver lipid accumulation was greater in those cows (Figure 1). Several factors preclude a direct comparison of our feed-restriction model with data obtained from peripartal cows. Around calving, adipose tissue is more sensitive to lipolytic agents, whereas insulin action is attenuated. Factors that improve insulin sensitivity, such as upregulated ADIPOR2 expression, appear to 
be an important adaptation to NEB in peripartal cows (Loor et al., 2006), but perhaps not during short-term feed restriction in mid lactation.

In dairy cattle, the final step of endogenous carnitine synthesis only occurs in the liver and kidney via $B B O X 1$ (Vaz and Wanders, 2002). Consistent with the stimulatory effect of thyroid hormone on lipid metabolism and CPT-1 activity (Louet et al., 2001), thyroxine administration increased hepatic $B B O X 1$ expression, BBOX1 activity, and carnitine accumulation (Galland et al., 2002). In contrast to early postpartal cows (Schlegel et al., 2012), we observed a decrease in the expression of BBOX1 (Figure 2) with feed restriction, suggesting a decrease in endogenous synthesis of carnitine. A moderate DMI depression in postpartal dairy cows was associated with greater hepatic carnitine concentration and a higher ratio of triiodothyronine to total thyroxine (Grum et al., 1996), suggesting a mechanistic link between these molecules. Acute feed restriction and the onset of lactation decrease serum triiodothyronine and thyroxine concentrations (Grum et al., 1996; Capuco et al., 2001); a lower thyroid hormone concentration in our feed-restricted cows might have affected $B B O X 1$ expression.

\section{Carbohydrate Metabolism During Feed Restriction}

The functional analysis of the microarray data did not suggest gluconeogenesis being an important adaptation; however, the increase expression of $P C$ and $P D K 4$ with qPCR in restricted- versus ad libitum-fed cows (Figure 2), key genes in gluconeogenesis, suggests an overall greater flux of alanine and lactate to oxaloacetate rather than acetyl-CoA. This can be a consequence of lower serum insulin in feed-restricted versus ad libitum cows (Carlson et al., 2006) in order to maintain similar glucose concentrations during feed restriction under NEB (Kuhla et al., 2009).

Partitioning of lactate and alanine toward gluconeogenesis is under the control of $P C$. Greater PC activity in periods of NEB allows pyruvate carbon to be channeled through oxaloacetate to maintain hepatic glucose output (Reynolds et al., 2003) as observed during the peripartal period (Greenfield et al., 2000; Loor et al., 2006), another feed restriction study in dairy cows (Velez and Donkin, 2005), and experimentally induced glucose deficit (Bradford and Allen, 2005). Thus, this enzyme seems to have flexibility in terms of its activation as a counter regulatory mechanism during periods of undernutrition.

Inactivation of $P D H$ is an essential control mechanism that spares glucose (and glucose precursors) during periods of undernutrition and NEB (Buck et al., 2002). Activity of $P D H$ is inhibited by allosteric effectors (acetyl-CoA and NADH) and phosphorylation by PDK4 (Sugden and Holness, 2003) and, thus, minimizes carbohydrate oxidation by preventing the flow of glycolytic products into the tricarboxylic acid cycle. The expression of $P D H B$ was reduced in feed-restricted cows (Supplemental Table S2). In a previous study a decrease in abundance of several glycolytic proteins was observed in response to feed deprivation in lactating dairy cows (Kuhla et al., 2009). In our study we did not observe changes in expression of genes coding for proteins directly involved in glycolysis or gluconeogenesis (Supplemental Table S2). Therefore, the increase in gluconeogenesis is essentially suggested by the upregulation of $P C$ and $P D K 4$ and downregulation of $P D H B$. However, the increase in gluconeogenic substrates, and therefore flux, is also supported by the overall lower in vitro alanine oxidation in liver from restricted- versus ad libitum-fed cows observed (Figure 1).

Interestingly, the increase in $\mathrm{PDK}_{4}$ expression by feed restriction in our experiment appeared to be partly inhibited by supplementation of L-carnitine (Figure 2). This is consistent to what observed in murine heart ventricles, where underfeeding increased expression of PDK4 and addition of L-carnitine administration prevented such increase (Horiuchi et al., 1999). In the same study, the carnitine-deficient mice had a greater increase in $P D K 4$ expression when underfed compared with wild-type mice. The apparent inhibition of PDK4 upregulation by L-carnitine suggested a lower degree of $P D H$ inhibition, thus, a likely greater use of glucose for energy production. Our data are consistent with the greater in vitro conversion of glucose to $\mathrm{CO}_{2}$ and a lack of increase in glucose production in cows supplemented with L-carnitine (Carlson et al., 2006). Overall, the data provided further support for a greater flux toward gluconeogenesis in feed-restricted cows but did not indicate a gluconeogenic effect of L-carnitine.

\section{Intracellular Energy Status and Oxidative Stress}

The inhibition of oxidative phosphorylation during feed restriction appears to be a consistent response in the liver of dairy cows (Loor et al., 2007; Kuhla et al., 2009). The same response was observed in rats (Rasheed et al., 1980); however, in chickens, transcripts related to oxidative phosphorylation were coordinately upregulated during starvation for 48 h (Desért et al., 2008). In our study, the reduction of mitochondrial respiratory activity during feed restriction was likely controlled by a large network of transcription factors, but primarily via the downregulation of the transcription factors $P H B$ and $N Q O 1$, which control the expression of BLVRB, CYB5B, NDUFA12, and NDUFS3. Those genes are related with oxidative phosphorylation and 
the electron transport chain (Supplemental Table S2 and Supplemental Figure S1). The observed reduction of protein expression of electron acceptors $(C Y B 5 B$ and $B L V R B)$ during feed restriction in lactating dairy cows supports previous observations (Kuhla et al., 2009). Furthermore, a decrease in CYB5B also would have indirectly reduced desaturation via $S C D$ (Kuhla et al., 2009). This observation, together with a large decrease in expression of $S C D$ (Table 2), suggests a decrease of unsaturated FA in liver and in circulation.

Besides the well-established adaptations in cellular metabolism, previous work also has demonstrated that gene expression changes due to undernutrition and NEB result in the alterations in hepatic signal transduction and host defense systems (Loor et al., 2007; McCarthy et al., 2010). Greater feed efficiency in growing cattle is a result of the combination of lower visceral organ weights (e.g., the liver), altered basal metabolism, improved mitochondrial electron transport, and reduced oxidative stress (Connor et al., 2010), whereas, feed deprivation diminishes the capability of the liver to counteract oxidative stress (Kuhla et al., 2009). Undernutrition can increase reactive oxygen metabolites, which may lead to the development of oxidative stress when produced in quantities that overwhelm the body's antioxidant defenses and predispose cows to disease (Bertoni et al., 2009). Insufficient energy intake leads to a fall in mitochondrial proton leak, which is positively related with reactive oxygen metabolite production (Brookes, 1998; Rezapour and Taghinejad-Roudbaneh, 2011).

In feed-restricted cows, the increase in GPX3 and SAA3 (Table 1) was associated with downregulated expression of genes involved in antioxidant defense, such as GSR and NQO1 (Supplemental Figure S3 and Supplemental Table S2). Those data were indicative of oxidative stress and inflammation. The promoter region of GPX3 contains an antioxidant response element and metal response element, which are known to be responsive to reactive oxygen metabolites (Dalton et al., 1996; Bierl et al., 2004). It is likely that the source of reactive oxygen metabolites in feed-restricted cows was from oxidation of incoming NEFA as a result of fat mobilization (Loor et al., 2007; Bertoni et al., 2009) coupled with the inhibition of oxidative phosphorylation (Brookes, 1998), and this elicited the increase in GPX3 expression observed.

\section{CONCLUSIONS}

Feed restriction and the resulting NEB altered the hepatic transcriptome in a way that suggested a reduction in the biosynthesis of cholesterol. In contrast, it activated key gluconeogenic genes, indicating an increase in the use of acetyl-CoA for gluconeogenesis instead of energy production. Similarly, the data indicated an increase in the flux of FA toward oxidation due to few key genes instead of a change in the transcription of several genes. Similar to peripartal cows, our data suggested that feed restriction in mid-lactation reduced overall metabolism and energy generation in the liver and enhanced glutathione metabolism to counteract oxidative stress. Liver tissue remodeling in mid-lactation also appears sensitive to undernutrition, as shown by genes associated with cell growth or proliferation, gene expression, and cell death. The induction of a proinflammatory state evidenced by changes in gene expression with feed restriction could be associated at least in part with greater liver TAG.

\section{REFERENCES}

Arkell, R. S., R. J. Dickinson, M. Squires, S. Hayat, S. M. Keyse, and S. J. Cook. 2008. DUSP6/MKP-3 inactivates ERK1/2 but fails to bind and inactivate ERK5. Cell. Signal. 20:836-843.

Bauer, M., A. C. Hamm, M. Bonaus, A. Jacob, J. Jaekel, H. Schorle, M. J. Pankratz, and J. D. Katzenberger. 2004. Starvation response in mouse liver shows strong correlation with life-span-prolonging processes. Physiol. Genomics 17:230-244.

Bertoni, G., E. Trevisi, and R. Lombardelli. 2009. Some new aspects of nutrition, health conditions and fertility of intensively reared dairy cows. Ital. J. Anim. Sci. 8:491-518.

Bierl, C., B. Voetsch, R. C. Jin, D. E. Handy, and J. Loscalzo. 2004. Determinants of human plasma glutathione peroxidase $(\mathrm{GPx}-3)$ expression. J. Biol. Chem. 279:26839-26845.

Bionaz, M., and J. J. Loor. 2012. Ruminant metabolic systems biology: reconstruction and integration of transcriptome dynamics underlying functional responses of tissues to nutrition and physiological state. Gene Regul. Syst. Bio. 6:109-125.

Bionaz, M., K. Periasamy, S. L. Rodriguez-Zas, W. L. Hurley, and J. J. Loor. 2012. A novel dynamic impact approach (DIA) for functional analysis of time-course omics studies: Validation using the bovine mammary transcriptome. PLoS ONE 7:e32455.

Bobe, G., J. W. Young, and D. C. Beitz. 2004. Invited review: Pathology, etiology, prevention, and treatment of fatty liver in dairy cows. J. Dairy Sci. 87:3105-3124.

Bradford, B. J., and M. S. Allen. 2005. Phlorizin administration increases hepatic gluconeogenic enzyme mRNA abundance but not feed intake in late-lactation dairy cows. J. Nutr. 135:2206-2211.

Brookes, P. S. 1998. Mitochondrial proton leak and superoxide generation: An hypothesis. Biochem. Soc. Trans. 26:S331.

Brumby, P. E., M. Anderson, B. Tuckley, J. E. Storry, and K. G. Hibbit. 1975. Lipid metabolism in the cow during starvation-induced ketosis. Biochem. J. 146:609-615.

Buck, M. J., T. L. Squire, and M. T. Andrews. 2002. Coordinate expression of the PDK4 gene: A means of regulating fuel selection in a hibernating mammal. Physiol. Genomics 8:5-13.

Butler, W. R., and R. D. Smith. 1989. Interrelationships between energy balance and postpartum reproductive function in dairy cattle. J. Dairy Sci. 72:767-783.

Bykov, I., H. Jarvelainen, and K. Lindros. 2003. L-Carnitine alleviates alcohol-induced liver damage in rats: Role of tumour necrosis factor-alpha. Alcohol Alcohol. 38:400-406.

Capuco, A. V., D. L. Wood, T. H. Elsasser, S. Kahl, R. A. Erdman, C. P. Van Tassell, A. Lefcourt, and L. S. Piperova. 2001. Effect of somatotropin on thyroid hormones and cytokines in lactating dairy cows during ad libitum and restricted feed intake. J. Dairy Sci. 84:2430-2439.

Carlson, D. B., N. B. Litherland, H. M. Dann, J. C. Woodworth, and J. K. Drackley. 2006. Metabolic effects of abomasal L-carnitine 
infusion and feed restriction in lactating Holstein cows. J. Dairy Sci. 89:4819-4834.

Carlson, D. B., J. W. McFadden, A. D'Angelo, J. C. Woodworth, and J. K. Drackley. 2007a. Dietary L-carnitine affects periparturient nutrient metabolism and lactation in multiparous cows. J. Dairy Sci. 90:3422-3441.

Carlson, D. B., J. C. Woodworth, and J. K. Drackley. 2007b. Effect of L-carnitine infusion and feed restriction on carnitine status in lactating Holstein cows. J. Dairy Sci. 90:2367-2376.

Collard, B. L., P. J. Boettcher, J. C. Dekkers, D. Petitclerc, and L. R. Schaeffer. 2000. Relationships between energy balance and health traits of dairy cattle in early lactation. J. Dairy Sci. 83:2683-2690.

Connor, E. E., S. Kahl, T. H. Elsasser, J. S. Parker, R. W. Li, C. P. Van Tassell, R. L. Baldwin, and S. M. Barao. 2010. Enhanced mitochondrial complex gene function and reduced liver size may mediate improved feed efficiency of beef cattle during compensatory growth. Funct. Integr. Genomics 10:39-51.

Cummins, C. L., D. H. Volle, Y. Zhang, J. G. McDonald, B. Sion, A. M. Lefrancois-Martinez, F. Caira, G. Veyssiere, D. J. Mangelsdorf, and J. M. A. Lobaccaro. 2006. Liver X receptors regulate adrenal cholesterol balance. J. Clin. Invest. 116:1902-1912. PubMed.

Dalton, T. P., Q. W. Lio, D. Bittel, L. C. Liang, and G. K. Andrews, 1996. Oxidative stress activates metal-responsive transcription factor-1 binding activity - Occupancy in vivo of metal response elements in the metallothionein-I gene promoter. J. Biol. Chem. 271:26233-26241.

Dann, H. M., and J. K. Drackley. 2005. Carnitine palmitoyltransferase I in liver of periparturient dairy cows: Effects of prepartum intake, postpartum induction of ketosis, and periparturient disorders. J. Dairy Sci. 88:3851-3859.

Désert, C., M. J. Duclos, P. Blavy, F. Lecerf, F. Moreews, C. Klopp, M. Aubry, F. Herault, P. Le Roy, C. Berri, M. Douaire, C. Diot, and S. Lagarrigue. 2008. Transcriptome profiling of the feeding-tofasting transition in chicken liver. BMC Genomics 9:611.

Drackley, J. K., H. M. Dann, G. N. Douglas, N. A. J. Guretzky, N. B. Litherland, J. P. Underwood, and J. J. Loor. 2005. Physiological and pathological adaptations in dairy cows that may increase susceptibility to periparturient diseases and disorders. Ital. J. Anim. Sci. 4:323-344

Galland, S., B. Georges, F. Le Borgne, G. Conductier, J. V. Dias, and J. Demarquoy. 2002. Thyroid hormone controls carnitine status through modifications of gamma-butyrobetaine hydroxylase activity and gene expression. Cell. Mol. Life Sci. 59:540-545.

Go, G. W., and A. Mani. 2012. Low-density lipoprotein receptor (LDLR) family orchestrates cholesterol homeostasis. Yale J. Biol. Med. 85:19-28

Greenfield, R. B., M. J. Cecava, and S. S. Donkin. 2000. Changes in mRNA expression for gluconeogenic enzymes in liver of dairy cattle during the transition to lactation. J. Dairy Sci. 83:1228-1236.

Grum, D. E., J. K. Drackley, R. S. Younker, D. W. LaCount, and J. J. Veenhuizen. 1996. Nutrition during the dry period and hepatic lipid metabolism of periparturient dairy cows. J. Dairy Sci. 79:1850-1864.

Horiuchi, M., K. Kobayashi, M. Masuda, H. Terazono, and T. Saheki 1999. Pyruvate dehydrogenase kinase $4 \mathrm{mRNA}$ is increased in the hypertrophied ventricles of carnitine-deficient juvenile visceral steatosis (JVS) mice. Biofactors 10:301-309.

Hosack, D. A., G. Dennis, Jr., B. T. Sherman, H. C. Lane, and R. A. Lempicki. 2003. Identifying biological themes within lists of genes with EASE. Genome Biol. 4:R70.

Huang, D. W., B. T. Sherman, and R. A. Lempicki. 2009. Systematic and integrative analysis of large gene lists using DAVID bioinformatics resources. Nat. Protoc. 4:44-57.

Hutchinson, I. A., A. A. Hennessy, S. M. Waters, R. J. Dewhurst, A. C. Evans, P. Lonergan, and S. T. Butler. 2012. Effect of supplementation with different fat sources on the mechanisms involved in reproductive performance in lactating dairy cattle. Theriogenology $78: 12-27$.

Ji, H., T. M. Bradley, and G. C. Tremblay. 1996. Atlantic salmon (Salmo salar) fed L-carnitine exhibit altered intermediary metabo- lism and reduced tissue lipid, but no change in growth rate. J. Nutr. 126:1937-1950.

Kuhla, B., D. Albrecht, S. Kuhla, and C. C. Metges. 2009. Proteome analysis of fatty liver in feed-deprived dairy cows reveals interaction of fuel sensing, calcium, fatty acid, and glycogen metabolism. Physiol. Genomics 37:88-98.

Liu, J., M. B. Rone, and V. Papadopoulos. 2006. Protein-protein interactions mediate mitochondrial cholesterol transport and steroid biosynthesis. J. Biol. Chem. 281:38879-38893.

Loor, J. J., H. M. Dann, R. E. Everts, R. Oliveira, C. A. Green, N. A. Guretzky, S. L. Rodriguez-Zas, H. A. Lewin, and J. K. Drackley. 2005. Temporal gene expression profiling of liver from periparturient dairy cows reveals complex adaptive mechanisms in hepatic function. Physiol. Genomics 23:217-226.

Loor, J. J., H. M. Dann, N. A. J. Guretzky, R. E. Everts, R. Oliveira, C. A. Green, N. B. Litherland, S. L. Rodriguez-Zas, H. A. Lewin, and J. K. Drackley. 2006. Plane of nutrition prepartum alters hepatic gene expression and function in dairy cows as assessed by longitudinal transcript and metabolic profiling. Physiol. Genomics 27:29-41.

Loor, J. J., R. E. Everts, M. Bionaz, H. M. Dann, D. E. Morin, R. Oliveira, S. L. Rodriguez-Zas, J. K. Drackley, and H. A. Lewin. 2007. Nutrition-induced ketosis alters metabolic and signaling gene networks in liver of periparturient dairy cows. Physiol. Genomics 32:105-116.

Louet, J. F., C. Le May, J. P. Pegorier, J. F. Decaux, and J. Girard. 2001. Regulation of liver carnitine palmitoyltransferase I gene expression by hormones and fatty acids. Biochem. Soc. Trans. 29:310-316.

McCarthy, S. D., S. M. Waters, D. A. Kenny, M. G. Diskin, R. Fitzpatrick, J. Patton, D. C. Wathes, and D. G. Morris. 2010. Negative energy balance and hepatic gene expression patterns in highyielding dairy cows during the early postpartum period: A global approach. Physiol. Genomics 42:188-199.

Owen, K. Q., H. Ji, C. V. Maxwell, J. L. Nelssen, R. D. Goodband, M. D. Tokach, G. C. Tremblay, and S. I. Koo. 2001. Dietary Lcarnitine suppresses mitochondrial branched-chain keto acid dehydrogenase activity and enhances protein accretion and carcass characteristics of swine. J. Anim. Sci. 79:3104-3112.

Poderoso, C., P. Maloberti, A. Duarte, I. Neuman, C. Paz, F. Cornejo Maciel, and E. J. Podesta. 2009. Hormonal activation of a kinase cascade localized at the mitochondria is required for StAR protein activity. Mol. Cell. Endocrinol. 300:37-42.

Rasheed, B. K., S. Chhabra, and C. K. Kurup. 1980. Influence of starvation and clofibrate administration on oxidative phosphorylation by rat liver mitochondria. Biochem. J. 190:191-198.

Reddy, J. K., and M. S. Rao. 2006. Lipid metabolism and liver inflammation. II. Fatty liver disease and fatty acid oxidation. Am. J. Physiol. Gastrointest. Liver Physiol. 290:G852-G858.

Reynolds, C. K., P. C. Aikman, B. Lupoli, D. J. Humphries, and D. E. Beever. 2003. Splanchnic metabolism of dairy cows during the transition from late gestation through early lactation. J. Dairy Sci. 86:1201-1217.

Rezapour, A., and M. Taghinejad-Roudbaneh. 2011. Effects of food restriction on oxidative stress indices in Ghezel ewes. J. Anim. Vet. Adv. 10:980-986.

Schlegel, G., J. Keller, F. Hirche, S. Geissler, F. J. Schwarz, R. Ringseis, G. I. Stangl, and K. Eder. 2012. Expression of genes involved in hepatic carnitine synthesis and uptake in dairy cows in the transition period and at different stages of lactation. BMC Vet. Res. 8:28.

Singh, R. P., P. Dhawan, C. Golden, G. S. Kapoor, and K. D. Mehta. 1999. One-way cross-talk between p38(MAPK) and p42/44(MAPK). Inhibition of p38(MAPK) induces low density lipoprotein receptor expression through activation of the p42/44(MAPK) cascade. J. Biol. Chem. 274:19593-19600.

Spaniol, M., P. Kaufmann, K. Beier, J. Wuthrich, M. Torok, H. Scharnagl, W. Marz, and S. Krahenbuhl. 2003. Mechanisms of liver steatosis in rats with systemic carnitine deficiency due to treatment with trimethylhydraziniumpropionate. J. Lipid Res. 44:144-153. 
Sugden, M. C., and M. J. Holness. 2003. Recent advances in mechanisms regulating glucose oxidation at the level of the pyruvate dehydrogenase complex by PDKs. Am. J. Physiol. Endocrinol. Metab. 284:E855-E862.

Supek, F., M. Bosnjak, N. Skunca, and T. Smuc. 2011. REVIGO summarizes and visualizes long lists of Gene Ontology terms. PLoS ONE 6:e21800.

Vaz, F. M., and R. J. Wanders. 2002. Carnitine biosynthesis in mammals. Biochem. J. 361:417-429.
Velez, J. C., and S. S. Donkin. 2005. Feed restriction induces pyruvate carboxylase but not phosphoenolpyruvate carboxykinase in dairy cows. J. Dairy Sci. 88:2938-2948.

Viturro, E., M. Koenning, A. Kroemer, G. Schlamberger, S. Wiedemann, M. Kaske, and H. H. D. Meyer. 2009. Cholesterol synthesis in the lactating cow: Induced expression of candidate genes. J. Steroid Biochem. Mol. Biol. 115:62-67. 